\title{
SOME INVARIANT PROPERTIES OF CURVES IN THE TAXICAB GEOMETRY
}

\author{
IDRIS ŐREN AND H. ANIL COBAN
}

\begin{abstract}
Let $E_{T}^{2}$ be the group of all isometries of the 2-dimensional taxicab space $R_{T}^{2}$. For the taxicab group $E_{T}^{2}$, the taxicab type of curves is introduced. All possible taxicab types are found. For every taxicab type, an invariant parametrization of a curve is described. The $E_{T}^{2}$-equivalence of curves is reduced to the problem of the $E_{T^{-}}^{2}$ equivalence of paths.
\end{abstract}

\section{INTRODUCTION}

The 2-dimensional taxicab space can be introduced using the metric $d_{T}(x, y)=\left|x_{1}-y_{1}\right|+\left|x_{2}-y_{2}\right|$ instead of the well-known Euclidean metric $d_{E}(x, y)=\left[\left(x_{1}-y_{1}\right)^{2}+\left(x_{2}-y_{2}\right)^{2}\right]^{\frac{1}{2}}$, where $x=\left(x_{1}, x_{2}\right), y=\left(y_{1}, y_{2}\right) \in R^{2}$. This space will be denoted by $R_{T}^{2}$. $R_{T}^{2}$ is also known as the taxicab plane geometry (shortly, taxicab geometry) $[9,10]$.

Let $E_{T}^{2}=\left\{F: R_{T}^{2} \rightarrow R_{T}^{2}: F x=g x+b, g \in D_{4}, b \in R_{T}^{2}\right\}$, where the group $D_{4}$ is the (Euclidean) symmetry group of the square.

The 2-dimensional taxicab group is introduced in [14]. For $n \geq 2$, geometric properties in the $n$-dimensional taxicab space are investigated in $[1,2,6,11]$. The taxicab arc length of a curve in the 2-dimensional taxicab space is defined in [17].

Invariant parametrizations and global properties of curves and paths in some spaces are considered in papers $[3,8,12,13]$ and some books $[5,7]$. Similar problems for taxicab geometry have not yet appeared in the literature. These results are important for the theory of curves, the problems of $E_{T}^{2}$-equivalence of curves and some physical applications. For example, the taxicab geometry plays an important role in ecology, firespread simulation with square-cell, grid-based maps $[4,15,18]$. Non-linear differential equations in taxicab geometry are introduced in [16].

This paper is organized as follows: In Section 2, the definitions of taxicab curve, taxicab type and the taxicab arc length function of a curve is given. In section 3, the definition of an invariant parametrization of a curve are given. Invariant parametrization of a curve with a fixed taxicab type are 
described. In Theorem 3.6, the problems of the $E_{T}^{2}$-equivalence of curves are reduced to that of paths.

Future research could include problems and applications concerning $E_{T^{-}}^{2}$ equivalence of curves as well as the complete system of differential invariants of a curve in $R_{T}^{2}$.

\section{The Taxicab Type of a Curve}

Let $R$ be the field of real numbers and $I=(a, b)$ an open interval of $R$.

Definition 2.1. A $C^{\infty}$ mapping $x: I \rightarrow R_{T}^{2}$ will be called an $I$-path (shortly, a path) in $R_{T}^{2}$.

Definition 2.2. An $I_{1}$-path $x(t)$ and an $I_{2}$-path $y(r)$ in $R_{T}^{2}$ will be called $D$-equivalent if a $C^{\infty}$-diffeomorphism $\varphi: I_{2} \rightarrow I_{1}$ exists such that $\varphi^{\prime}(r)>0$ and $y(r)=x(\varphi(r))$ for all $r \in I_{2}$. A class of $D$-equivalent paths in $R_{T}^{2}$ will be called a curve in $R_{T}^{2}$. A path $x \in \alpha$ will be called a parametrization of a curve $\alpha$.

We denote the group $\left\{F: R_{T}^{2} \rightarrow R_{T}^{2}: F x=g x+b, g \in D_{4}, b \in R_{T}^{2}\right\}$ of all transformations of $R_{T}^{2}$ by $E_{T}^{2}$, where $g x$ is the multiplication of a matrix $g$ and a column vector $x \in R_{T}^{2}$.

If $x(t)$ is an $I$-path then $F x(t)$ is an $I$-path in $R_{T}^{2}$ for any $F \in E_{T}^{2}$. Let $G$ be a subgroup of $E_{T}^{2}$.

Definition 2.3. Two $I$-paths $x(t)$ and $y(t)$ in $R_{T}^{2}$ are called $G$-equivalent if there exists $F \in G$ such that $y(t)=F x(t)$. This being the case, we write $x(t) \stackrel{G}{\sim} y(t)$.

Let $\alpha=\left\{h_{\tau}, \tau \in Q\right\}$ be a curve in $R_{T}^{2}$, where $h_{\tau}$ is a parametrization of $\alpha$. Then $F \alpha=\left\{F h_{\tau}, \tau \in Q\right\}$ is a curve in $R_{T}^{2}$ for any $F \in E_{T}^{2}$.

Definition 2.4. Two curves $\alpha$ and $\beta$ in $R_{T}^{2}$ are called $G$-equivalent if $\beta=F \alpha$ for some $F \in G$. This being the case, we write $\alpha \stackrel{G}{\sim} \beta$.

Let $x(t)=\left(x_{1}(t), x_{2}(t)\right)$ be an $I$-path in $R_{T}^{2}, x^{\prime}(t)=\left(x_{1}^{\prime}(t), x_{2}^{\prime}(t)\right)$ be the derivative of the path $x(t)$. For $p, q \in I=(a, b), p<q$, we let

$$
l_{x}(p, q)=\int_{p}^{q}\left(\left|x_{1}^{\prime}(t)\right|+\left|x_{2}^{\prime}(t)\right|\right) d t .
$$

Obviously, the finite and infinite limits $l_{x}(a, q)=\lim _{p \rightarrow a} l_{x}(p, q) \leq+\infty$ and $l_{x}(p, b)=\lim _{q \rightarrow b} l_{x}(p, q) \leq+\infty$ exist. We have the following four possibilities:

$$
l_{x}(a, q)<+\infty, \quad l_{x}(p, b)<+\infty
$$




\section{INVARIANT PROPERTIES IN THE TAXICAB GEOMETRY}

$$
\begin{array}{ll}
l_{x}(a, q)<+\infty, & l_{x}(p, b)=+\infty \\
l_{x}(a, q)=+\infty, & l_{x}(p, b)<+\infty \\
l_{x}(a, q)=+\infty, & l_{x}(p, b)=+\infty .
\end{array}
$$

Suppose that the case (2.1) holds for some $p, q \in I$. Then $l=l_{x}(a, q)+$ $l_{x}(p, b)-l_{x}(p, q)$, where $0 \leq l \leq+\infty$, does not depend on $p, q \in I$. In this case we say that $x$ belongs to the taxicab type of $(0, l)$. In cases $(2.2)$, $(2.3)$, and (2.4), we say that $x$ has taxicab types $(0,+\infty),(-\infty, 0)$, and $(-\infty,+\infty)$, respectively. The taxicab type of a path $x$ will be denoted by $L(x)$.

Remark 2.5. The following examples 2.6-2.9 below show that there exist paths of all types $(0, l)$, where $l<+\infty,(0,+\infty),(-\infty, 0),(-\infty,+\infty)$.

Example 2.6. Consider the $I$-path $x(t)=($ rcost,rsint $)$ in $E_{T}^{2}$, where $I=(0, \pi / 2)$ and $r>0$. Then

$$
l_{x}(p, q)=r \int_{p}^{q}(\sin t+\cos t) d t=r(-\cos q+\sin q+\cos p-\sin p)
$$

for all $0<p<q<\frac{\pi}{2}$.

Since $l_{x}(0, q)=\lim _{p \rightarrow 0} l_{x}(p, q)<+\infty$ and $l_{x}\left(p, \frac{\pi}{2}\right)=\lim _{q \rightarrow \frac{\pi}{2}} l_{x}(p, q)<$ $+\infty$, the type of the path is $(0, l)$.

Example 2.7. Consider the $I$-path $x(t)=\left(t, e^{t}\right)$ in $E_{T}^{2}$, where $I=(0,+\infty)$. Then

$$
l_{x}(p, q)=\int_{p}^{q}\left(1+e^{t}\right) d t=q-p+e^{q}-e^{p}
$$

for all $0<p<q$.

Since $l_{x}(0, q)=\lim _{p \rightarrow 0} l_{x}(p, q)<+\infty$ and $l_{x}(p,+\infty)=\lim _{q \rightarrow+\infty} l_{x}(p, q)=$ $+\infty$, the type of the path is $(0,+\infty)$.

Example 2.8. Consider the $I$-path $x(t)=\left(t, t^{2}\right)$ in $E_{T}^{2}$, where $I=(-\infty, 0)$. Then

$$
l_{x}(p, q)=\int_{p}^{q}(1-2 t) d t=q-p-q^{2}+p^{2}
$$

for all $-\infty<p<q<0$.

Since $l_{x}(-\infty, q)=\lim _{p \rightarrow-\infty} l_{x}(p, q)=+\infty$ and $l_{x}(p, 0)=\lim _{q \rightarrow 0} l_{x}(p, q)<$ $+\infty$, the type of the path is $(-\infty, 0)$. 
Example 2.9. Consider the $I$-path $x(t)=\left(t, t^{2}\right)$ in $E_{T}^{2}$, where $I=(-\infty,+\infty)$. Then

$$
l_{x}(p, q)=\int_{p}^{q}(1+2|t|) d t=q-p+q^{2}+p^{2}
$$

for all $p<0<q$.

Since

$$
\begin{aligned}
l_{x}(-\infty, q) & =\lim _{p \rightarrow-\infty} l_{x}(p, q)=+\infty \\
\text { and } l_{x}(p,+\infty) & =\lim _{q \rightarrow \infty} l_{x}(p, q)=+\infty,
\end{aligned}
$$

the type of the path is $(-\infty,+\infty)$.

Proposition 2.10. Let $x(t)$ be an I-path in $R_{T}^{2}$. Then $l_{x}(p, q)=l_{g x}(p, q)$ for all $g \in D_{4}$

Proof. Since $x(t)$ is an $I$-path in $R_{T}^{2}, g x(t)$ is an $I$-path in $R_{T}^{2}$ for all $g \in D_{4}$. Since the derivative of the $I$-path $x(t)$ is $x^{\prime}(t)=\left(x_{1}^{\prime}(t), x_{2}^{\prime}(t)\right)$, we have $[g x(t)]^{\prime}=g x^{\prime}(t)$ for all $g \in D_{4}$ and for all $t \in I$. Then an $I$-path $g x^{\prime}(t)$ can be written in forms $\left(x_{1}^{\prime}(t), x_{2}^{\prime}(t)\right),\left(-x_{1}^{\prime}(t), x_{2}^{\prime}(t)\right),\left(x_{1}^{\prime}(t),-x_{2}^{\prime}(t)\right),\left(-x_{1}^{\prime}(t)\right.$, $\left.-x_{2}^{\prime}(t)\right),\left(x_{2}^{\prime}(t), x_{1}^{\prime}(t)\right),\left(-x_{2}^{\prime}(t), x_{1}^{\prime}(t)\right),\left(x_{2}^{\prime}(t),-x_{1}^{\prime}(t)\right),\left(-x_{2}^{\prime}(t),-x_{1}^{\prime}(t)\right)$. Clearly, $l_{x}(p, q)=l_{g x}(p, q)$ for all $g \in D_{4}$.

Corollary 2.11. Let $x(t)$ be an I-path in $R_{T}^{2}$. Then $l_{x}(p, q)=l_{F x}(p, q)$ for all $F \in E_{T}^{2}$.

Proof. It follows from Proposition 2.10.

Proposition 2.12. Let $x(t)$ and $y(t)$ be two I-paths in $R_{T}^{2}$. Then

(i) if $x \stackrel{E_{T}^{2}}{\sim} y$ then $L(x)=L(y)$.

(ii) if $x, y$ are parametrizations of a curve $\alpha$ then $L(x)=L(y)$.

Proof. It is obvious.

The taxicab type of a path $x \in \alpha$, will be called the taxicab type of the curve $\alpha$ and denoted by $L(\alpha) . L(\alpha)$ is an $E_{T}^{2}$-invariant of a curve $\alpha$.

Definition 2.13. An $I$-path $x(t)$ is called regular if $x^{\prime}(t) \neq 0$ for all $t \in I$.

If $x(t)$ is a regular path and a path $y(t)$ for all $t \in I$ is $D$-equivalent to $x(t)$, the $y(t)$ is also a regular path for all $t \in I$. A curve $\alpha$ is called regular if it contains a regular path. 


\section{INVARIANT PROPERTIES IN THE TAXICAB GEOMETRY}

\section{Invariant Parametrization of the Taxicab Curve}

Now we define an invariant parametrization of a regular curve in $R_{T}^{2}$. Let $I=(a, b)$ and $x(t)$ be a regular $I$-path in $R_{T}^{2}$. We define the taxicab arc length function $s_{x}(t)$ for each taxicab type as follows. We put $s_{x}(t)=$ $l_{x}(a, t)$ for the case $L(x)=(0, l)$, where $l \leq+\infty$, and $s_{x}(t)=-l_{x}(t, b)$ for the case $L(x)=(-\infty, 0)$. Let $L(x)=(-\infty,+\infty)$. We choose a fixed point in every interval $I=(a, b)$ of $R$ and denote it by $a_{I}$. Let $a_{I}=0$ for $I=(-\infty,+\infty)$. We set $s_{x}(t)=l_{x}\left(a_{I}, t\right)$.

Since $s_{x}^{\prime}(t)>0$ for all $t \in I$, the inverse function of $s_{x}(t)$ exists. Let us denote it by $t_{x}(s)$. The domain of $t_{x}(s)$ is $L(x)$ and $t_{x}^{\prime}(s)>0$ for all $s \in L(x)$.

Proposition 3.1. Let $I=(a, b)$ and $x$ be a regular $I$-path in $R_{T}^{2}$. Then

(i) $s_{F x}(t)=s_{x}(t)$ and $t_{F x}(s)=t_{x}(s)$ for all $F \in E_{T}^{2}$;

(ii) the equalities $s_{x(\varphi)}(r)=s_{x}(\varphi(r))+s_{0}$ and $\varphi\left(t_{x(\varphi)}\left(s+s_{0}\right)\right)=t_{x}(s)$ hold for any $C^{\infty}$-diffeomorphism $\varphi: J=(c, d) \rightarrow I$ such that $\varphi^{\prime}(r)>0$ for all $r \in J$, where $s_{0}=0$ for $L(x) \neq(-\infty,+\infty)$ and $s_{0}=l_{x}\left(\varphi\left(a_{J}\right), a_{I}\right)$ for $L(x)=(-\infty,+\infty)$.

Proof. The statement $(i)$ is obvious. Let us prove statement $(i i)$. Let $L(x)=(-\infty,+\infty)$. Then we have

$$
\begin{aligned}
s_{x(\varphi)}(r) & =\int_{a_{J}}^{r}\left(\left|\frac{d}{d r} x_{1}(\varphi(r))\right|+\left|\frac{d}{d r} x_{2}(\varphi(r))\right|\right) d r \\
& =\int_{a_{J}}^{r} \frac{d \varphi}{d r}\left(\left|\frac{d}{d \varphi} x_{1}(\varphi(r))\right|+\left|\frac{d}{d \varphi} x_{2}(\varphi(r))\right|\right) d r \\
& =l_{x}\left(\varphi\left(a_{J}\right), \varphi(r)\right)=l_{x}\left(a_{I}, \varphi(r)\right)+l_{x}\left(\varphi\left(a_{J}\right), a_{I}\right) .
\end{aligned}
$$

Thus, $s_{x(\varphi)}(r)=s_{x}(\varphi(r))+s_{0}$, where $s_{0}=l_{x}\left(\varphi\left(a_{J}\right), a_{I}\right)$. This implies that $\varphi\left(t_{x(\varphi)}\left(s+s_{0}\right)\right)=t_{x}(s)$. For $L(x) \neq(-\infty,+\infty)$, it is easy to see that $s_{0}=0$.

Let $\alpha$ be a regular curve, $x \in \alpha$. Then $x\left(t_{x}(s)\right)$ is a parametrization of $\alpha$.

Definition 3.2. The parametrization of the form $x\left(t_{x}(s)\right)$ of a regular curve $\alpha$ is called an invariant parametrization of $\alpha$.

Denote the set of all invariant parametrizations of $\alpha$ by $I_{p}(\alpha)$. Every $y \in I_{p}(\alpha)$ is a $J$-path, where $J=L(\alpha)$.

Proposition 3.3. Let $\alpha$ be a regular curve, $x \in \alpha$ and $x$ be a J-path, where $J=L(\alpha)$. Then the following conditions are equivalent: 
(i) $x$ is an invariant parametrization of $\alpha$;

(ii) $\left|x_{1}^{\prime}(s)\right|+\left|x_{2}^{\prime}(s)\right|=1$ for all $s \in L(\alpha)$;

(iii) $s_{x}(s)=s$ for all $s \in L(\alpha)$.

Proof. $(i) \rightarrow($ ii $)$. Let $x \in I_{p}(\alpha)$. Then there exists $y \in \alpha$ such that $x(s)=$ $y\left(t_{y}(s)\right)$. By Proposition 3.1, $s_{x}(s)=s_{y\left(t_{y}\right)}(s)=s_{y}\left(t_{y}(s)\right)+s_{0}=s+s_{0}$, where $s_{0}$ is as in Proposition 3.1. Since $s_{0}$ does not depend on $s$, we have $\frac{d s_{x}(s)}{d s}=\left|x_{1}^{\prime}(s)\right|+\left|x_{2}^{\prime}(s)\right|=1$ for all $s \in L(\alpha)$.

$($ ii $) \rightarrow($ iii $)$. Let $\left|x_{1}^{\prime}(s)\right|+\left|x_{2}^{\prime}(s)\right|=1$ for all $s \in L(\alpha)$. Using the definition of $s_{x}(t)$, we get $\frac{d s_{x}(s)}{d s}=\left|x_{1}^{\prime}(s)\right|+\left|x_{2}^{\prime}(s)\right|=1$. Therefore $s_{x}(s)=$ $s+c$ for some $c \in R$. In the case $L(x) \neq(-\infty,+\infty)$, conditions $s_{x}(s)=s+c$ and $s_{x}(s) \in L(\alpha)$ for all $s \in L(\alpha)$ implies $c=0$, that is, $s_{x}(s)=s$. In the case $L(\alpha)=(-\infty,+\infty)$, equalities $s_{x}(s)=l_{x}\left(a_{J}, s\right)=l_{x}(0, s)=s+c$ implies $0=l_{x}(0,0)=c$, that is, $s_{x}(s)=s$. $I_{p}(\alpha)$.

$($ iii $) \rightarrow(i)$. Since $s_{x}(s)=s$ implies $t_{x}(s)=s$, we get $x(s)=x\left(t_{x}(s)\right) \in$

Proposition 3.4. Let $\alpha$ be a regular curve and $L(\alpha) \neq(-\infty,+\infty)$. Then there exists a unique invariant parametrization of $\alpha$.

Proof. Let $x, y \in \alpha, x$ be an $I_{1}$-path. Then there exists a $C^{\infty}$ - diffeomorphism $\varphi: I_{2} \rightarrow I_{1}$ such that $\varphi^{\prime}(r)>0$ and $y(r)=x(\varphi(r))$ for all $r \in I_{2}$. By Proposition 3.3 and $L(\alpha) \neq(-\infty,+\infty)$, we obtain $y\left(t_{y}(s)\right)=x\left(\varphi\left(t_{y}(s)\right)=\right.$ $x\left(\varphi\left(t_{x(\varphi)}(s)\right)\right)=x\left(t_{x}(s)\right)$.

Proposition 3.5. Let $\alpha$ be a regular curve, $L(\alpha)=(-\infty,+\infty)$ and $x \in$ $I_{p}(\alpha)$. Then $I_{p}(\alpha)=\{y: y(s)=x(s+c), c \in(-\infty,+\infty)\}$.

Proof. Let $x, y \in I_{p}(\alpha)$. Then there exist $h, k \in \alpha$ such that $x(s)=$ $h\left(t_{h}(s)\right), y(s)=k\left(t_{k}(s)\right)$, where $h$ is an $I_{1}$-path and $k$ is an $I_{2}$-path. Since $h, k \in \alpha$ there exists $\varphi: I_{2} \rightarrow I_{1}$ such that $\varphi^{\prime}(r)>0$ and $k(r)=h(\varphi(r))$ for all $r \in I_{2}$. By Proposition 3.1,y $y(s)=k\left(t_{k}(s)\right)=h\left(\varphi\left(t_{k}(s)\right)=\right.$ $h\left(\varphi\left(t_{h(\varphi)}(s)\right)=h\left(t_{h}\left(s-s_{0}\right)\right)=x\left(s-s_{0}\right)\right.$.

Let $x \in I_{p}(\alpha)$ and $s^{\prime} \in(-\infty,+\infty)$. We proof $x(\theta) \in I_{p}(\alpha)$, where $\theta(s)=s+s^{\prime}$. By Proposition 3.3, $\left|x_{1}^{\prime}(s)\right|+\left|x_{2}^{\prime}(s)\right|=1$ and $s_{x}(s)=s$. Put $z(s)=x(\theta(s))$. Since $\theta$ is a $C^{\infty}$-diffeomorphism of $(-\infty,+\infty)$ onto $(-\infty,+\infty)$, then $z=x(\theta) \in \alpha$. Using Proposition 3.1 and $s_{x}(s)=s$, we get $s_{z}(s)=s_{x(\theta)}(s)=s_{x}(\theta(s))+s_{1}=\left(s+s^{\prime}\right)+s_{1}$, where

$$
s_{1}=\int_{\theta(0)}^{0}\left(\left|x_{1}^{\prime}(s)\right|+\left|x_{2}^{\prime}(s)\right|\right) d s
$$


for $s \in L(\alpha)$.

This, in view of $\left|x_{1}^{\prime}(s)\right|+\left|x_{2}^{\prime}(s)\right|=1$, implies $s_{1}=-\theta(0)=-s^{\prime}$. Then $s_{z}(s)=\left(s+s^{\prime}\right)-s^{\prime}=s$. By Proposition 3.3, $z \in I_{p}(\alpha)$.

Theorem 3.6. Let $\alpha, \beta$ be regular curves and $x \in I_{p}(\alpha), y \in I_{p}(\beta)$. Then

(i) for $L(\alpha)=L(\beta) \neq(-\infty,+\infty), \alpha \stackrel{E_{T}^{2}}{\sim} \beta$ if and only if $x \stackrel{E_{T}^{2}}{\sim} y$;

(ii) for $L(\alpha)=L(\beta)=(-\infty,+\infty), \alpha \stackrel{E_{T}^{2}}{\sim} \beta$ if and only if $x \stackrel{E_{T}^{2}}{\sim} y\left(\psi_{c}\right)$ for some $c \in(-\infty,+\infty)$, where $\psi_{c}(s)=s+c$.

Proof. (i). Let $\alpha \stackrel{E_{T}^{2}}{\sim} \beta$ and $h \in \alpha$. Then there exists $F \in E_{T}^{2}$ such that $\beta=F \alpha$. This implies $F h \in \beta$. Using Propositions 3.1-3.4, we get $x(s)=$ $h\left(t_{h}(s)\right), y(s)=(F h)\left(t_{F h}(s)\right)$ and $F x(s)=F\left(h\left(t_{h}(s)\right)\right)=(F h)\left(t_{h}(s)\right)=$ $(F h)\left(t_{F h}(s)\right)=y(s)$. Thus $x \stackrel{E_{T}^{2}}{\sim} y$. Conversely, let $x \stackrel{E_{T}^{2}}{\sim} y$, that is, there exists $F \in E_{T}^{2}$ such that $F x=y$. Then $\alpha \stackrel{E_{T}^{2}}{\sim} \beta$.

(ii). Let $\alpha \stackrel{E_{T}^{2}}{\sim} \beta$. Then there exist $J$-paths $h \in \alpha, k \in \beta$ and $F \in E_{T}^{2}$ such that $k(t)=F h(t)$. We have $k\left(t_{k}(s)\right)=k\left(t_{F h}(s)\right)=k\left(t_{h}(s)\right)=(F h)\left(t_{h}(s)\right)$. By Proposition 3.5, $x(s)=k\left(t_{k}\left(s+s_{1}\right)\right), y(s)=h\left(t_{h}\left(s+s_{2}\right)\right)$ for some $s_{1}, s_{2} \in(-\infty,+\infty)$. Therefore, $x\left(s-s_{1}\right)=F y\left(s-s_{2}\right)$. This implies that $x \stackrel{E_{T}^{2}}{\sim} y\left(\psi_{c}\right)$, where $\psi_{c}(s)=s+c$ and $c=s_{1}-s_{2}$. Conversely, let $x \stackrel{E_{T}^{2}}{\sim} y\left(\psi_{c}\right)$ for some $c \in(-\infty,+\infty)$, where $\psi_{c}=s+c$. Then there exists $F \in E_{T}^{2}$ such that $y(s+c)=F x(s)$. Since $y(s+c) \in \beta$, then $\alpha \stackrel{E_{T}^{2}}{\sim} \beta$.

Theorem 3.6 reduces problems of the $E_{T}^{2}$-equivalence regular curves to that of paths only for the case $L(\alpha)=L(\beta) \neq(-\infty,+\infty)$. Let $G$ be a subgroup of $E_{T}^{2}$.

Definition 3.7. $J$-paths $x(t)$ and $y(t)$ will be called

$[G,(-\infty,+\infty)]$-equivalent, if there exist $g \in G$ and $d \in(-\infty,+\infty)$ such that $y(t)=g x(t+d)$ for all $t \in J$.

Theorem 3.6 reduces problems of the $G$-equivalence of regular curves to $[G,(-\infty,+\infty)]$-equivalence of paths for the case $L(\alpha)=L(\beta)=(-\infty,+\infty)$.

\section{ACKNOWLEDGMENT}

The authors are very grateful to the referee for helpful comments and valuable suggestions.

\section{REFERENCES}

[1] Z. Akca and R. Kaya, On the distance formulae in three dimensional taxicab space, Hadronic Journal, 27.5 (2004), 521-532. 
[2] Z. Akca and R. Kaya, On the norm in higher dimensional taxicab spaces, Hadronic J. Suppl., 19 (2004), 491-501.

[3] R. G. Aripov and D. Khadjiev, The complete system of differential and integral invariants of a curve in Euclidean geometry, Russian Mathematics, 51.7 (2007), $1-14$

[4] D. Caballero, Taxicab geometry: some problems and solutions for square grid-based fire spread simulation, V. International Conference on Forest Fire Research, 2006.

[5] S. S. Chern, Curves and surfaces in Euclidean space, Global Diff. Geom., 27 (1989), 99-139.

[6] Ö. Gelisken and R. Kaya, The taxicab space group, Acta Math.Hungar., 122.1-2 (2009), 187-200

[7] D. Khadjiev, An Application of Invariant Theory to Differential Geometry of Curves, Fan Publ., Tashkent, 1988. [Russian]

[8] D. Khadjiev and Ö. Pekşen, The complete system of global integral and differential invariants for equi-Affine curves, Differ. Geom. Appl., (2004), no. 20, 167-175.

[9] E. F. Krause, Taxicab Geometry, Addison-Wesley, Menlo Park, 1975.

[10] K. Menger, You Will Like Geometry, Guidebook for Illinois Institute of Technology Geometry Exhibit, Museum of Science and Industry, Chicago, III., 1952.

[11] M. Özcan, S. Ekmekci, and A. Bayar, A note on the variation of taxicab length under rotations, Pi Mu Epsilon Journal, 11.7 (Fall 2002), 381-384.

[12] Ö. Pekșen, D. Khadjiev, and İ. Ören, Invariant parametrizations and complete systems of global invariants of curves in the pseudo-Euclidean geometry, Turk. J. Math., 36.1 (2012), 147-160.

[13] Y. Sagiroglu and Ö. Pekșen, The equivalence of centro-equi-affine curves, Turk. J. Math, 34 (2010), 95-104.

[14] D. J. Schattschneider, The taxicab group, Amer. Math. Monthly, 97.7 (1984), 423428.

[15] M. W. Sohn, Distance and cosine measures of niche overlap, Soc. Networks, 23 (2001), 141-165.

[16] R. A. Struble, Non-linear Differential Equations, McGraw-Hill Comp., New York, 1962 .

[17] K. P. Thompson, The nature of length, area, and volume in taxicab geometry, Int. Electron. J. Geom., 4.2 (2011), 193-207.

[18] D. L. Warren, R. E. Glor, and M. Turelli, Environmental niche equivalency versus conservatism: quantitative approaches to niche evolution, Evolution, 62.11 (2008), 2868-2883.

MSC2010: 51K05, 51K99, 51N30, 51F20, 53A55, 53A35

Key words and phrases: Curve, Taxicab geometry, Invariant parametrization

Department of Mathematics, Karadeniz Technical University, Faculty of Science, 61080 Trabzon, Turkey

E-mail address: orenidris@gmail.com

Department of Mathematics, Karadeniz Technical University, Faculty of Science, 61080 Trabzon, Turkey

E-mail address: hacoban@ktu.edu.tr 